\title{
MACROALGAS ADERIDAS EM RIZÓFOROS DE Rizophora mangle L. EM BOA VIAGEM-SÃO JOSÉ DE RIBAMAR-MARANHAO
}

\author{
Luina Santos Serra Freire ${ }^{1}$, Luana da Silva Viana², Natália de Jesus Carneiro Silva² \& Khey Albert de \\ Azevedo Fontes ${ }^{3 *}$
}

\begin{abstract}
'Discente do curso de Licenciatura em Ciências Agrárias, IFMA campus São Luís-Maracanã, Avenida dos Curiós, S/ №, Vila Esperança, São Luís-MA, CEP: 65095-460, bolsista IC-FAPEMA, luinafreire@outlook.com
\end{abstract}

²Discentes do curso de Licenciatura em Ciências Agrárias, IFMA campus São Luís-Maracanã, Avenida dos Curiós,
S/N $N^{\circ}$, Vila Esperança, São Luís-MA, CEP: 65095-460, luanaviana692@gmail.com, carneiro_001@hotmail.com

${ }^{3}$ Instituto Federal de Educação, Ciência e Tecnologia do Maranhão IFMA Campus São Luís-Maracanã, Avenida dos Curiós, S/No , Vila Esperança, São Luís-MA, CEP: 65095-460, autor correspondente: khey.fontes@ifma.edu.br

\section{RESUMO}

Esta abordagem teve como objetivo analisar as comunidades de macroalgas aderidas em rizóforos de Rizophora mangle L. na praia de Boa Viagem, São José de Ribamar-Maranhão como parâmetro para compreender melhor os diferentes níveis de variação observados no "Bostrychietum" de áreas distintas de manguezal nas duas baías que compõem o Golfão Maranhense. Foram traçados 12 transectos de linha entre novembro de 2018 e maio de 2019. Foram analisados 108 rizóforos englobados pelos transectos ao longo das coletas. Foram registrados os valores de $\mathrm{O}_{2}$ dissolvido, temperatura da água, $\mathrm{pH}$ e salinidade, bem como foram considerados os valores de precipitação disponibilizados por órgão oficial. 17 taxa infragenéricos (06 Chlorophyta e 11 Rhodophyta) foram identificados. Observou-se um crescimento tanto na biomassa total quanto na biomassa das espécies ao longo das coletas. Não foi observado nenhum padrão determinante de distribuição vertical das macroalgas. Os valores de precipitação apresentaram uma correlação positiva com os valores de biomassa algácea total.

Palavras-chave: algas, manguezal, "Bostrychietum"

\begin{abstract}
This survey aimed to assess macroalgae community attached to rhizophores of Rizophora. mangle L. in Boa Viagem beach, São José de Ribamar, Maranhão, as a parameter to a better understand of the different levels of variation observed inside "Bostrychietum" from different areas of mangroves in the two bays that integrate Golfão Maranhense. 12 line transects were performed from november/2018 to may/2019. 108 rhizophores selected randomly along the transects were analised. Values of dissolved $\mathrm{O}_{2}$, water temperature, $\mathrm{pH}$ and salinity were registered as well as precipitation levels, provided by official department, were also considered. 17 infrageneric taxa were identified (06 Chlorophyta and 11 Rhodophyta). It has been observed an increase in total biomass, as well as individual biomass species along the time of sampling. Vertical distribution pattern were not observed. Precipitation levels correlated positively to total macroalgae biomass.

Key words: algae, mangrove, "Bostrychietum"
\end{abstract}

\section{INTRODUÇÃO}

As macroalgas que compõem o "Bostrychietum" (Post, 1936) podem variar em ocorrência, frequência e biomassa de uma área de manguezal para outra. Além disso, essa variação pode sofrer influência significativa dos ecossistemas adjacentes como praias rochosas, recifes, dentre outros (Taylor, 1960; Oliveira, 1984). Embora observa-se a predominância de Rhodophyta como os gêneros Bostrychia Mont., Caloglossa (Harv.) G. Martens e Catenella Grev. (Pedroche et al. 1995), algumas Chlorophyta como os gêneros Cladophoropsis Borgesen e Rhizoclonium
Kutzing (Fernandes et al. 2005; Fontes et al. 2016; Cutrim \& Azevedo, 2005) também podem ocorrer e apresentar valores de biomassa e frequência igualmente consideráveis.

Os substratos utilizados para fixação das macroalgas no manguezal são representados principalmente por troncos, rizóforos e pneumatóforos das angiospermas constituintes do mangue como Rhizophora mangle L. e Avicennia germinans (L.) Stearn, porém, eventuais poças e remanescentes rochosos, quando presentes, podem também ser utilizados pela comunidade algácea para habitar o 
referido ecossistema (Oliveira, 1984). Assim sendo, a composição do "Bostrychietum" pode apresentar níveis significativos de variação numa mesma área dependendo da disponibilidade de substrato. Algumas espécies podem também apresentar um nível de predileção por determinado substrato (Eston et al. 1992 ), mesmo que essa relação ainda não esteja claramente elucidada.

Varias abordagens importantes vem, ao longo do tempo, indicando a grande importância das macroalgas de manguezal, tanto como componente da biodiversidade como na análise de aspectos ecológicos elucidativos como a contribuição de sua biomassa para a produção primária do ecossistema (Pedroche et al. 1995; Pereira, 2000; Cunha \& Costa, 2002;). Vários indicadores apontam para o valor biológico destas comunidades como também sugerem o potencial inerente às espécies no que diz respeito ao conhecimento e uso sustentável de suas características economicamente viáveis.

A Ilha de São Luís é completamente envolvida por áreas de manguezais que se localizam nas duas baías que a circundam (São Marcos e São José) e que compõem o Golfão Maranhense. Apesar de apresentarem propriedades em comum, como amplitudes de maré de aproximadamente 8 metros (Coutinho \& Morais, 1976; EMAP, 2020), as duas baías demonstram características distintas que poderiam ser capazes de interferir na composição algácea do "Bostrychietum" das respectivas áreas de manguezal. Além disso, a existência de um complexo portuário ativo localizado na baía de São Marcos poderá ocasionar impactos nessas comunidades algáceas, contribuindo para as variações na diversidade. Portanto, o estudo da composição das macroalgas em áreas diferentes localizadas nas duas baías poderá permitir a avaliação de critérios tanto para compreender a diversidade algácea como também a ser utilizados em processos de monitoramento ambiental e outras aplicações, pois, estudos anteriores indicam grande importância ecológica (Pereira, 2000; Miranda \& Pereira, 1989), bem como elevada utilidade como ferramenta de monitoramento do ambiente (Fontes et al. 2007).

Com o objetivo de compreender melhor os diferentes níveis de variação observados no "Bostrychietum" de diferentes áreas de manguezal nas duas baías que compõem o Golfão Maranhense, este trabalho analisou as comunidades de macroalgas na praia de Boa Viagem, São José de RibamarMaranhão, através da composição e distribuição sazonal das espécies em rizóforos de Rizophora mangle L. bem como relacionou estes critérios com a variação sazonal de parâmetros ambientais importantes para o desenvolvimento das macroalgas registrada no referida estação de coletas.

\section{MATERIAL E MÉTODOS}

O manguezal da Praia de Boa Viagem, São José de Ribamar, MA (02³4'55.99''S; 4405'35.70”O) localiza-se a nordeste da Ilha de São Luís, na Baía de S. José. Caracteriza-se por ser um remanescente de manguezal de fácil acesso, sem impactos evidentes e com bosque composto pelas espécies de angiospermas Avicennia germinans (L.) Steam e Rhizophora mangle (L.). Os substratos disponíveis (pneumatóforos, rizóforos e troncos) apresentam evidente presença de macroalgas (Fontes et al. 2015) (Figura. 1).

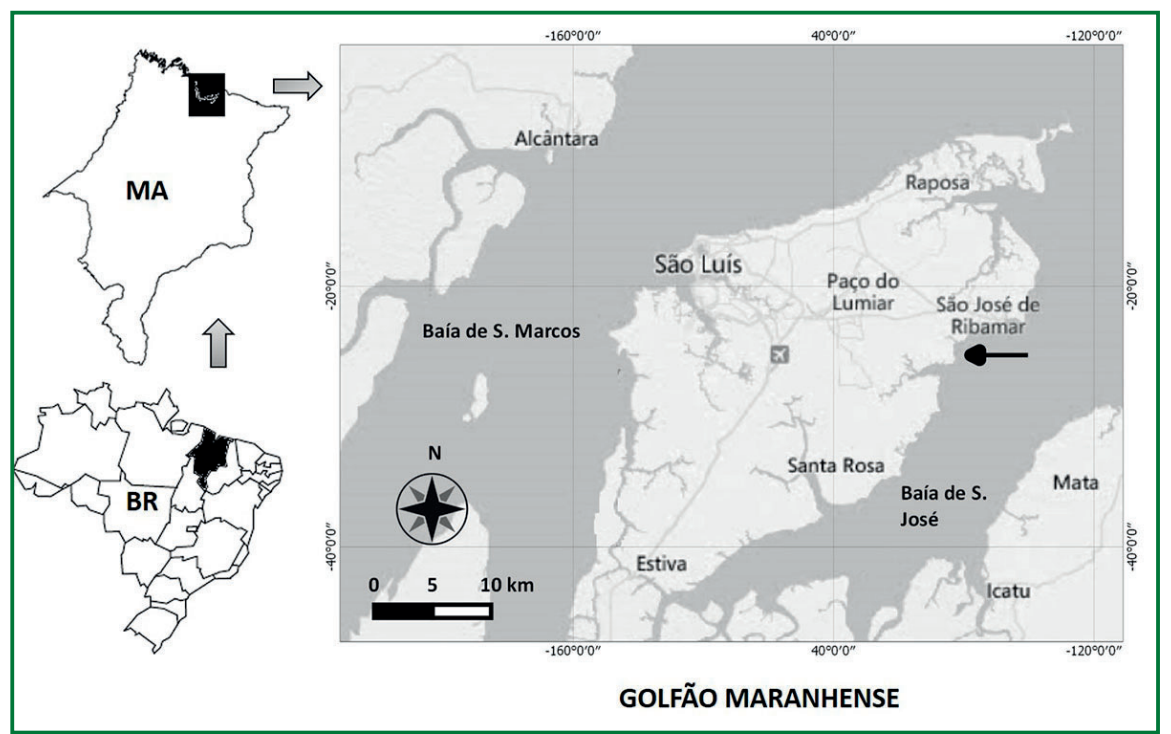

Figura 1. Área de estudos. Praia de Boa Viagem, São José de Ribamar, MA (seta). Fonte: Fontes et al. 2015. 
Foram traçados três transectos de linha sequenciados e eqüidistantes entre si, cuja distância foi calculada de acordo com o comprimento total da área de estudo, modificado de Davey e Woelkerling (1985). As coletas foram realizadas em 08/11/2018 (C1), 06/12/2018 (C2), correspondendo ao período de estiagem, 30/04/2019 (C3) e 15/05/2019 (C4) correspondendo ao período chuvoso. Cada transecto correspondeu ao comprimento de cinco metros e foi traçado, conforme distância calculada, em uma região próxima à terra firme (subárea 3 ), próxima à entrada da maré (subárea 1) e em uma região intermediária (subárea 2). Em cada transecto foram escolhidos três indivíduos de $R$. mangle e em cada indivíduo foram escolhidos três rizóforos com evidente presença de macroalgas totalizando 108 rizóforos analisados. Cada rizóforo foi seccionado em três secções de altura denominadas secção $1(0-15 \mathrm{~cm})$, secção $2(15-30 \mathrm{~cm})$ e secção $3(30-45 \mathrm{~cm})$ com os limites correspondendo à cobertura algácea total em relação ao sedimento. O material algal ocorrente nos rizóforos foi coletado com uma espátula, devidamente acondicionados em sacos plásticos em seguida e levados ao laboratório para análise posterior.

No Laboratório de Botânica Aquática, LABOTAq do IFMA Campus São Luís-Maracanã a identificação das macroalgas baseou-se na morfologia externa e interna do talo com o auxílio de microscópios estereoscópico e óptico. Para a confirmação das espécies analisadas foram consultadas bibliografias pertinentes. A posição taxonômica dos taxa foi organizada segundo Wynne (2017).

A freqüência das macroalgas nos rizóforos foi obtida através da razão entre o número de rizóforos em que a macroalga ocorreu e o número total de rizóforos a ser analisado multiplicado por 100 , modificado de Fontes et al.(2007).

Para facilitar o entendimento da freqüência de ocorrência, foi adotada uma tabela de valores seguintes (Davey \& Woelkerling, 1985):

$>75 \%=$ espécie muito freqüente

$75 \mid-50 \%=$ espécie freqüente

$50 \mid-25 \%=$ espécie pouco freqüente

$\leq 25 \%=$ espécie rara

Imediatamente após a identificação e análise da frequência de ocorrência de cada macroalga, o material algal foi levado à estufa a uma temperatura de $\pm 75^{\circ} \mathrm{C}$ para que se promovesse a total desidratação até que se alcançasse o peso constante, correspondente ao valor de sua biomassa.

As espécies de Rhizoclonium Kutz. e Pseudorhizoclonium (Kutz.) Boedeker, quando presentes, foram identificadas, contudo, sua biomassa foi calculada e considerada como Complexo RHZ, visto que a triagem a fim de se obter a biomassa separada não é considerada viável (Cutrim et al. 2004; Fontes et al. 2007).

O nível de oxigênio $(\mathrm{O} 2)$ dissolvido na água, a temperatura da água, bem como os valores de $\mathrm{pH}$ foram registrados in loco através de oxímetro a cada coleta. A salinidade foi medida in loco através de salinômetro. Os valores de precipitação foram disponibilizados pelo Laboratório de Meteorologia LABMET do Núcleo Geoambiental da Universidade Estadual do Maranhão, UEMA.

Para analisar a relação entre o aumento da biomassa algácea total e o aumento da precipitação ao longo dos períodos de coleta foi utilizado a Correlação Linear de Pearson através do software BioEstat 5.3.

\section{RESULTADOS}

A Tabela 1 apresenta as espécies de macroalgas ocorrentes nos rizóforos analisados na praia de Boa Viagem em São José de Ribamar, Ma. Foram identificadas 17 taxa infragenéricos, seis Chlorophyta (Caulerpa fastigiata Montagne; Cladophora vagabunda (Linnaeus) Hoek; Cladophoropsis membranacea (Hofman Bang ex C.Agardh) Borgesen; Pseudorhizoclonium africanum (Kützing) Boedeker; Rhizoclonium riparium (Roth) Harvey e Ulva sp) e 11 Rhodophyta (Bostrychia binderi Harvey; Bostrychia calliptera (Montagne) Montagne; Bostrychia montagnei Harvey; Bostrychia moritziana (Sonder ex Kützing) J.Agardh; Bostrychia radicans (Montagne) Montagne; Caloglossa leprieurii (Montagne) G.Martens; Caloglossa ogasawaraensis Okamura; Catenella caespitosa (Withering) L.M.Irvine; Murrayella periclados (C.Agardh) F.Schmitz; Polysiphonia howei Hollenberg e Polysiphonia subtilissima Montagne.

Todas as espécies de Bostrychia identificadas neste estudo registraram altos valores de frequência $(100 \%)$ sendo classificadas como espécies muito frequentes (MF) nos rizóforos estudados. $C$. leprieurii, C. caespitosa, C. membranacea e P. howei, $P$. africanum $\mathrm{e}$. riparium também registraram altos valores de frequência e também foram consideradas muito frequentes $(100 \%, \mathrm{MF})$. P. subtilíssima registrou valores médios de frequência $(50 \%)$ e foi a única espécie considerada como frequente (F) nos rizóforos estudados. C. ogasawaraensis, C. fastigiata, C. vagabunda e Ulva sp. registraram baixos valores de frequência $(25 \%)$ e foram consideradas como 
espécies raras (R). Todas as espécies raras foram coletadas em coletas únicas, C. ogasawaraensis e C. vagabunda em $\mathrm{C}_{3}$, C. fastigiata em $\mathrm{C}_{2}$ e Ulva sp. em $\mathrm{C}_{1}$ (Tabela 1).

A biomassa algácea total registrada ao longo do período de coletas apresentou valores crescentes de $\mathrm{C}_{1}$ a $\mathrm{C}_{4}$ com pequena variação em $\mathrm{C}_{2}$ (Figura 2A). Essa tendência de crescimento da biomassa pode ser observada também quando as espécies foram consideradas individualmente (Figura 2B2D). B. calliptera apresentou maior biomassa nos períodos de coleta quando comparada tanto com as outras espécies do gênero quanto com as outras espécies ocorrentes (Figura 2B) contribuindo com $73,23 \%$ para a constituição da biomassa algácea total (Tabela 1). Entre as demais espécies ocorrentes de Bostrychia, B. radicans e B. montagnei destacam-se por terem registrado elevados valores de biomassa, contribuindo em $5,93 \%$ e $5,12 \%$, respectivamente, para a biomassa total das algas (Figura 2C; Tabela 1).

C. leprieurii, C. caespitosa e C. membranacea foram as demais espécies ocorrentes que registraram valores significativos de biomassa ao longo dos períodos de coleta, mesmo quando comparadas com os valores mais elevados de biomassa registrados pelas espécies do gênero Bostrychia. As referidas espécies, porém, contribuíram pouco para a constituição da biomassa total algácea $(1,73 \%$, $3,39 \%$ e $2,02 \%$, respectivamente). O complexo RHZ, mesmo registrando valores muito baixos de biomassa quando comparado com os valores registrados para as demais espécies ocorrentes, apresentou valores relativamente estáveis durante todo o período de coletas, contudo, contribuiu pouco com a biomassa total $(0,90 \%)$ (Figura 2D; Tabela 1). Ulva sp. registrou valor de biomassa inferior ao erro padrão da balança analítica usada na obtenção da biomassa e sua indicação na Tabela 1 intenciona apenas ocorrência esporádica da espécie, não sendo, assim, considerada para a biomassa algácea total.

Os valores de biomassa total registrados apresentaram crescimento nas três secções dos rizóforos ao longo das quatro coletas realizadas neste estudo. A secção 1 , correspondente à variação de altura do rizóforo entre 0 e $15 \mathrm{~cm}$, registrou maior biomassa algácea total, enquanto que a secção 3 , correspondente ao intervalo de altura entre 30 e 45 $\mathrm{cm}$, registrou a menor (Figura 3A). A tendência de crescimento da biomassa total das algas também foi observada nas subáreas delimitadas pelos transectos

Tabela 1. Macroalgas ocorrentes nos rizóforos de $R$. mangle L. ao longo dos períodos de coletas $(\mathrm{C} 1$, C2, C3 e C4) na praia de Boa Viagem, São José de Ribamar, Ma. * presença; - ausência; $\downarrow$ menor que $0,0005 \mathrm{~g}$ (erro da balança de precisão); F.O frequência de ocorrência; Bt biomassa total (g/m-2); Cbt contribuição da espécie para a biomassa total.

\begin{tabular}{|c|c|c|c|c|c|c|c|}
\hline Espécie & $\mathrm{C}_{1}$ & $\mathrm{C}_{2}$ & $\mathrm{C}_{3}$ & $\mathrm{C}_{4}$ & F.O & Bt & Cbt \\
\hline Bostrychia binderi Harvey & $*$ & $*$ & $*$ & $*$ & $100 \% \mathrm{MF}$ & 3.315 & $1,95 \%$ \\
\hline Bostrychia calliptera (Montagne) Montagne & $*$ & * & * & * & $100 \% \mathrm{MF}$ & 124.193 & $73,23 \%$ \\
\hline Bostrychia montagnei Harvey & $*$ & * & * & $*$ & $100 \% \mathrm{MF}$ & 8.688 & $5,12 \%$ \\
\hline $\begin{array}{l}\text { Bostrychia moritziana (Sonder ex Kützing) } \\
\text { J.Agardh }\end{array}$ & $*$ & $*$ & $*$ & $*$ & $100 \% \mathrm{MF}$ & 5.045 & $2,97 \%$ \\
\hline Bostrychia radicans (Montagne) Montagne & $*$ & * & * & * & $100 \% \mathrm{MF}$ & 10.068 & $5,93 \%$ \\
\hline Caloglossa leprieurii (Montagne) G.Martens & $*$ & * & $*$ & $*$ & $100 \% \mathrm{MF}$ & 2.950 & $1,73 \%$ \\
\hline Caloglossa ogasawaraensis Okamura & - & - & $*$ & - & $25 \% \mathrm{R}$ & 0,022 & $0,01 \%$ \\
\hline Catenella caespitosa (Withering) L.M.Irvine & $*$ & * & $*$ & $*$ & $100 \% \mathrm{MF}$ & 5.761 & $3,39 \%$ \\
\hline Caulerpa fastigiata Montagne & - & $*$ & - & - & $25 \% \mathrm{R}$ & 0,241 & $0,14 \%$ \\
\hline Cladophora vagabunda (Linnaeus) Hoek & - & - & $*$ & - & $25 \% \mathrm{R}$ & 0,766 & $0,45 \%$ \\
\hline $\begin{array}{l}\text { Cladophoropsis membranacea (Hofman Bang ex } \\
\text { C.Agardh) Borgesen }\end{array}$ & $*$ & $*$ & * & $*$ & $100 \% \mathrm{MF}$ & 3.431 & $2,02 \%$ \\
\hline Murrayella periclados (C.Agardh) F.Schmitz & $*$ & * & * & $*$ & $100 \% \mathrm{MF}$ & 1.952 & $1,15 \%$ \\
\hline Polysiphonia howei Hollenberg & - & * & * & $*$ & $75 \% \mathrm{MF}$ & 0,033 & $0,02 \%$ \\
\hline Polysiphonia subtilissima Montagne & - & - & $*$ & $*$ & $50 \% \mathrm{~F}$ & 0,019 & $0,01 \%$ \\
\hline Pseudorhizoclonium africanum (Kützing) Boedeker & $*$ & * & $*$ & $*$ & $100 \% \mathrm{MF}$ & 1.535 & $0,90 \%$ \\
\hline Rhizoclonium riparium (Roth) Harvey & $*$ & * & * & $*$ & $100 \% \mathrm{MF}$ & 1.535 & $0,90 \%$ \\
\hline Ulva sp & $*$ & - & - & - & $\mathrm{R}$ & $\downarrow$ & - \\
\hline
\end{tabular}



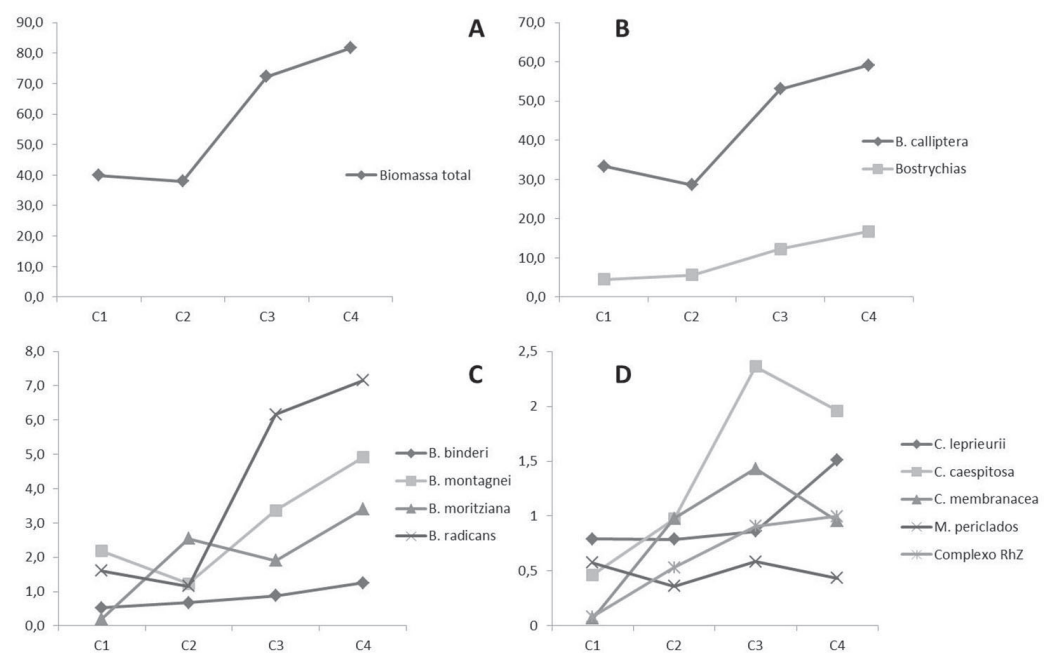

Figura 2. Variação da biomassa algácea $\left(\right.$ g. $\left.\mathrm{m}^{-2}\right)$ em valores totais das espécies de macroalgas ocorrentes em rizóforos de R. mangle L. na praia de Boa Viagem, São José de Ribamar, Ma. A) biomassa total. B) B. calliptera x "Bostrychias". C) Espécies de Bostrychia. D) Demais espécies ocorrentes.

A

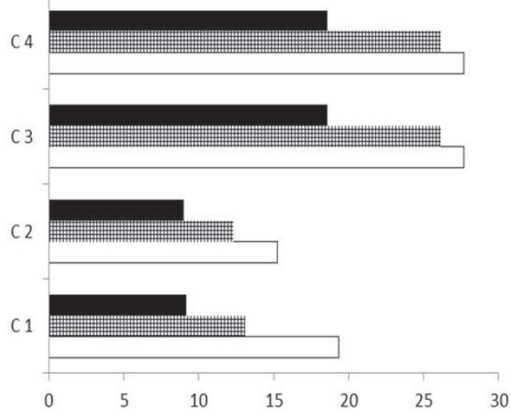

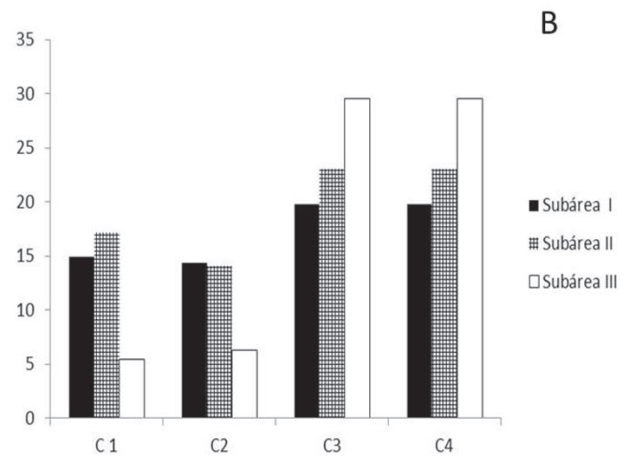

Figura 3. Variação da biomassa total $\left(\mathrm{g} \cdot \mathrm{m}^{-2}\right)$ das macroalgas aderidas em rizóforos de $R$. mangle L. na praia de Boa Viagem, São José de Ribamar, MA. A) Biomassa por secção de rizóforo; B) Biomassa por subárea de transecto.
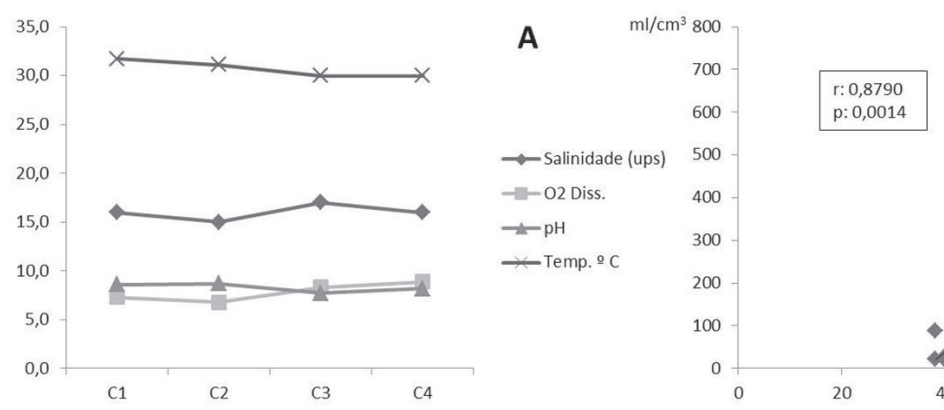

B

Figura 4. A)Valores das variantes ambientais registrados ao longo do período das coletas na praia de Boa Viagem, São José de Ribamar, MA. B) Correlação positiva entre os valores de biomassa algácea total e de precipitação obtida através do cálculo do Coeficiente de Correlação de Pearson (r). 
traçados. A subárea 3, mais próxima da terra firma, registrou maior biomassa algácea enquanto que a subárea 1 , mais próxima da orla, registrou valores mais baixos ao longo das coletas (Figura 3B).

Os valores dos fatores ambientais permaneceram relativamente constantes ao longo das coletas (Figura 4A). O Coeficiente de Correlação de Pearson usado para relacionar o aumento da biomassa algácea total com o aumento da precipitação ao longo do período de coletas demonstrou que há uma correlação positiva entre estes parâmetros no manguezal estudado (Figura 4B).

\section{DISCUSSÃO}

A riqueza de espécies encontrada nos rizóforos estudados assemelha-se com a riqueza de espécies registrada para outras áreas de manguezal tanto em nível mundial (Melville et al. 2005; Pedroche et al. 1995), quanto em nível de áreas brasileiras acessadas anteriormente (Paula et al. 1989; Cutrim \& Azevedo, 2005; Fontes et al. 2007). A elevada ocorrência de espécies do gênero Bostrychia caracteriza a comunidade de macroalgas como "Bostrychietum" proposta por Post (1936). A ocorrência de C. leprieurii e C. caespitosa contribui de forma positiva para a caracterização desta referida comunidade algácea.

Quando comparadas com a ocorrência das espécies de macroalgas em rizóforos de manguezais localizados na baía de São Marcos, MA (Cutrim et al. 2004) as espécies ocorrentes neste estudo corresponderam tanto em riqueza quanto em variação. Além disso, a existência do complexo portuário ativo localizado na baía de São Marcos parece não influenciar diretamente na composição dos componentes do "Bostrychietum" das áreas de manguezal localizadas nesta baía, considerando a correspondência de espécies. Contudo, esta relação deve ser monitorada mais detalhadamente em abordagens futuras.

Fontes et al. (2016) ao realizarem uma abordagem de composição, frequência e biomassa para macroalgas aderidas em pneumatóforos no mesmo manguezal, observou que $B$. radicans também foi considerada espécie muito frequente no referido substrato, assim como $B$. binderi foi considerada espécie rara em pneumatóforos. Ainda na mesma abordagem os autores consideraram a alta frequência de espécies constituintes do complexo RHZ e de macroalgas associadas como C. lepreurii e C. caespitosa. Tais fatos contribuem para que as comunidades de macroalgas tanto em rizóforos quanto em pneumatóforos sejam consideradas semelhantes em frequência de ocorrência na área estudada.

$\mathrm{O}$ aumento de biomassa das macroalgas ao longo do período das coletas registrado neste estudo também pode ser observado em outras abordagens (Cutrim \& Azevedo, 2007; Fernandes et al. 2005), muito embora os dados não apresentem natureza conclusiva. A expressividade de B. calliptera tanto em frequência quanto em biomassa, caracterizando esta espécie como o principal componente do "Bostrychietum" em rizóforos na praia de Boa Viagem, também foi observada por Cutrim et al. (2004) quando acessaram áreas de manguezal na Baía de São Marcos. Fontes et al. (2016) não observaram a ocorrência da referida espécie, indicando $B$. radicans como espécie mais expressiva nos pneumatóforos da mesma área.

Apesar de não ter sido observado nenhum padrão definido de distribuição vertical das macroalgas, visto que as espécies ocorreram por toda a extensão dos rizóforos, o registro do aumento gradativo da biomassa da secção 3 (mais alta) à secção 1(mais baixa) pode refletir o nível de tolerância à dessecação das referidas macroalgas (Mann \& Steinke, 1988), uma vez que, no manguezal de Boa Viagem, foi observado que as macroalgas aderidas nas porções de rizóforos mais próximas do sedimento estão constantemente em contato com poças de água temporárias. A tolerância à dessecação também foi considerado determinante por Cutrim et al (2004) para explicar a distribuição das espécies de macroalgas nos manguezais de Parna-Açu e TauáMirim na baía de São Marcos.

Se considerarmos o aumento da biomassa algácea total da subárea I (menor valor registrado) à subárea 3 (maior valor registrado), observaremos um padrão de crescimento que também já foi observado por Cutrim et al. (2004). Este padrão se mostra sensível à extensão da área, uma vez que manguezais com margem extensa são propensos a demonstrar distribuição horizontal das macroalgas (Fontes et al. 2007; Laursen \& King, 2000). Entretanto, Fontes et al. (2015) não observaram padrão definido de distribuição horizontal quando analisaram as macroalgas aderidas em pneumatóforos no mesmo manguezal.

Embora os valores das variantes ambientais registrados não tenham sido suficientes para esclarecer a influência desses fatores na variação da comunidade de macroalgas tanto em frequência quanto em biomassa, é de suma importância ressaltar que a tendência de crescimento pode ser afetada 
em vários níveis por determinadas características ambientais (Fontes et al. 2007; Melville et al. 2005). A correlação positiva entre os valores de biomassa total e de precipitação no período das coletas sugerem que os índices pluviométricos parecem ter maior influência no crescimento da biomassa algácea do que os outros fatores considerados neste Estudo.

\section{AGRADECIMENTOS}

Os autores agradecem à FAPEMA - Fundação de Amparo a Pesquisa e ao Desenvolvimento Cientifico e Tecnológico do Maranhão pela bolsa concedida, ao Instituto Federal de Educação, Ciência e Tecnologia do Maranhão - IFMA - Campus São Luís - Maracanã pelo apoio no desenvolvimento da abordagem e ao Laboratório de Meteorologia - LABMET do Núcleo Geoambiental da Universidade Estadual do Maranhão, UEMA pelos dados disponibilizados.

\section{REFERÊNCIAS}

BIOESTAT 5.3. Download de Arquivos. Disponível em:http://www.mamiraua.org.br/ download/index.phpdirpath=./BioEstat $\% 205 \% 20$ Portugues\&order=0. Acesso em: 14 mar 2019.

COUTINHO, P.N. \& MORAIS, J.O. 1976. Distribuição de sedimentos na Baía de São José, Estado do Maranhão (Brasil). Arquivo de Ciências do Mar, 16(2): 123-127.

CUNHA, S.R. \& COSTA, C.S.B. 2002. Gradientes de salinidade e freqüência de alagamento como determinantes da distribuição e biomassa de macroalgas associadas a troncos de manguezais na Baía de Babitonga, SC. Notas Técnicas Facimar, 6: 93-102.

CUTRIM, M.V.J., SILVA, E.F. \& AZEVEDO, A.C.G. 2004. Distribuição vertical das macroalgas aderidas em rizóforos de Rhizophora mangle Linnaeus nos manguezais de Parna-Açu e Tauá-Mirim (Ilha de São Luís/MA - Brasil). Boletim do Laboratório de Hidrobiologia, 17: 9-18.

CUTRIM, M.V. J. \& AZEVEDO, A.C.G. 2005. Macroalgas. Pp. 53-79. In: M. E. B. Fernandes (org.). Os manguezais da costa norte brasileira 2. Maranhão, Fundação Rio Bacanga

DAVEY, A. \& WOELKERLING, W.M.J. 1985. Studies on Australian mangrove algae. III. Victorian communities: struture and recolonization in Western Port Bay. Journal of Experimental Marine Biology and Ecology, 85: 177-190.
E M P R E A M A R A N H E N E D E ADMINISTRAÇÃO PORTUÁRIA, EMAP. Disponível em: http://www. portodoitaqui.ma.gov. br/porto-do-itaqui/localizacao . Acesso em: 29 de março de 2020.

ESTON, V.R., BRAGA, M.R.A., CORDEIROMARINO, M., FUJI, M.T. \& YOKOTA, N.S. 1992. Macroalgal colonization patterns on artificial substrates inside southeastern Brazilian mangroves. Aquatic Botany, 42: 315-325.

FERNANDES, M.E.B., SILVA, E.F., LIMA, J.F., VARELA, E.S., HERCOS, A.P., FERNANDES, C.M., ARRUDA, C.C.B., GOMES, G., SANTOS, H., SOARES, C. \& SARAIVA, R.M. 2005. Distribuição especial de macroalgas associadas às florestas de mangue na Península de Ajuruteua, Bragança-Pará. Boletim do Laboratório de Hidrobiologia, 18: 11-17.

FONTES, K.A.A., PEREIRA, S.M.B. \& ZICKEL, C.S. 2007. Macroalgas do "Bostrychietum" aderido em pneumatóforos de duas áreas de manguezal do Estado de Pernambuco. Iheringia - Série Botânica, 62: 31-38.

FONTES, K.A.A., LISBOA, A.T. \& CASTRO, R.S. 2016. Macroalgas aderidas em pneumatóforos de Avicennia germinans (L.) Stearn na praia de Boa Viagem, São José de Ribamar-Maranhão. Acta Tecnológica, 10(2): 61-73.

LAURSEN, W.J. \& KING, R.J. 2000. The distribution and abundance of macroalgae in Woolooware Bay, New South Wales, Australia. Botanica Marina, 43: 377-384.

MANN, F.D. \& STEINKE, T.D. 1988. Photosynthetic and respiratory responses of the mangrove-associated red algae, Bostrychia radicans and Caloglossa leprieurii. South. Africa. Journal of Botany, 54: 203-207.

MELVILLE, F., PULKOWNIK, A. \& BURCHETT, M. 2005. Zonal and seasonal variation in the distribution and abundance of mangrove macroalgae in the Parramatta River, Australia. Estuarine, Coastal and Shelf Science,64: 267-276.

MIRANDA, P.T.C. \& PEREIRA, S.M.B. 1989. Macroalgas bentônicas no manguezal do rio Ceará (Ceará-Brazil). 11- Distribuição em função das condições hidrológicas. Arquivos de Ciência do Mar, 28: 39-52.

OLIVEIRA, E.C. 1984. Brazilian mangal vegetation with special emphasis on the seaweeds. Pp. 55-65. In: POR, F.D. \& DOR, I. (org.) Hyrobiology of the 
mangal. Dr. W. Junk Publishers, The Hague.

PAULA; E.J., UGADIM, Y. \& KANAGAWA, A.I. 1989. Macroalgas de manguezais da Ilha de Maracá - Estado do Amapá, Brasil. Insula, 19: 95-114.

PEDROCHE, F.F., WEST, J.A., ZUCCARELLO, G.C., SENTÍES, A.G. \& KARSTEN, U. 1995. Marine red algae of the mangroves in Southern Pacific México and Pacific Guatemala. Botanica Marina, 38: 111-119.

PEREIRA, S.M.B. 2000. As algas bentônicas. In: BARROS, H.M., ESKINAZI-LEÇA, E., MACEDO, S.J. \& LIMA, T. (Ed.) Gerenciamento Participativo de Estuários e Manguezais. Recife: Ed. Universitária da Universidade Federal de Pernambuco. p. 49-65. POST, E. 1936. Systematische und pflanzengeographische Notizen zur BostrychiaCaloglossa Assoziation. Revist Algologik, 9: 1-84.

TAYLOR, W.R. 1960. Marine algae of the Eastern Tropical and Subtropical coasts of the Americas. Ann. Arbor: University of Michigan Press. 870 p.

WYNNE, M.J. 2017. A checklist of benthic marine algae of the tropical and subtropical western Atlantic: fourth revision. Nova Hedwigia, 145: 1-202. 